\title{
The Milky Way Comes from Hydrogen Fusion Big Bang
}

\section{YinYue Sha*}

Dongling Engineering Center, Ningbo Institute of Technology, Zhejiang University, PR China

We know that humans have created the atomic bomb and hydrogen bomb, the atomic bomb need high purity of uranium 235, in contrast, uranium 238 cannot be used to make an atomic bomb. The most important is not how much internal nucleon number of the nucleus, but the internal structure of stability, with nuclear structure consisting of four nucleuses is a special stability, but instead of two stable nuclear group together to become unstable nuclear structure, and four nuclear group of nuclear structure also has the very high stability [1]. From the nucleus losses mass in the picture can be seen that the most stable elements is in the near 64 and iron.

The hydrogen bomb is much more energy than the atom bomb, but without the atom bomb, the hydrogen bomb wouldn't explode anyway. In short, wood carbon is burned, but it is hard to burn without a light fire.

Today, mankind has created the atomic bomb and hydrogen bomb today, we can put two problems: make an atomic bomb from uranium is how form? If there is only one kind element of hydrogen in the world, how do you make hydrogen fusion?

The atomic bomb and uranium element on earth existence has been the fact that can't deny, the earth is a planet in the solar system, the orbits of planets in the solar system exist the systematic rule, as a result, the earth was produced during the formation of the solar system, not from outside the solar system.

In the process of the formation of the solar system, a large number of objects into the center of the solar system, planets have attracted a large number of objects, so, from the composition of the material, the planets and the sun is no big difference [2]. Uranium is found even small planet earth, in the condition of the earth can't spontaneous formation of uranium, the uranium element existing on earth, also can't deny the sun have uranium.

From the elements, the stability of the helium 4 is very stable, oxygen 16 is also stable, and near iron elements of the system is stable elements, so that the heavy nuclear fission will become to the near iron elements, light nuclear fusion becomes to the near iron elements, whether in the fusion and fission produces a lot of energy, hot enough to melt all matters.
According to fluid dynamics, the high density matters sinking, low density matters floating, low melting point matters melt [3]. In this way, in the original state of high temperature, high density and high melting point material formed the center of the sun, the gas matter is leaved from the sun by radiation energy, low temperature gas matters cooling effect on the planet, in this way, small celestial bodies are more likely to cooling and solidification and become solid.

Small objects are attracted to large bodies by large objects, thus forming a systematic structure of the distribution of celestial bodies: from the object as a whole, center high density, outer low density, due to the small bodies attracted to large objects fall into large objects, so the big objects the local existence of dense material.

The earth's early days were fireballs, and the earth's atmosphere and water were mostly from the sun.

Exists on the sun and the earth almost matter, as a result, people in the understanding of the sun out of the question, the sun is not composed of hydrogen and hydrogen is found in most of the material in the solar atmosphere, the core inside the sun is large number of high density of elements, the nuclear reaction on the sun is heavy nuclear fission, the sun overall density is similar to the overall density of the earth, as a result, human underestimated the density of the sun.

If the original sun is composed of hydrogen, just the mass of the sun can't make hydrogen fusion reaction, for hydrogen nuclear fusion mass are 228279339125 solar masses, namely the mass of the galaxy. The Milky Way come from hydrogen fusion big bang, the sun is just one of many stars in the Milky Way, and not too close to the center of the Milky Way, the earth also can't stand too close to the sun, otherwise, there would be no life on earth.

\section{References}

1. Tattersall JJ (2005) Elementary number theory in nine chapters. Cambridge University Press, UK.

2. Erica K (2013) Unheralded Mathematician Bridges the Prime Gap. Simons Science News, China.

3. Sha YY (2016) Polignac's Conjecture with New Prime Number Theorem. J Phys Math 7: 201.
*Corresponding author: YinYue Sha, Dongling Engineering Center, Ningbo Institute of Technology, Zhejiang University, PR China, Tel: 5718517 2244; E-mail: shayinyue@qq.com

Received June 19, 2017; Accepted June 27, 2017; Published June 30, 2017

Citation: Sha YY (2017) The Milky Way Comes from Hydrogen Fusion Big Bang J Biom Biostat 8: 352. doi: 10.4172/2155-6180.1000352

Copyright: @ 2017 Sha YY. This is an open-access article distributed under the terms of the Creative Commons Attribution License, which permits unrestricted use, distribution, and reproduction in any medium, provided the original author and source are credited. 